\title{
Mean Field Methods for Cortical Network Dynamics
}

\author{
John Hertz ${ }^{1}$, Alexander Lerchner ${ }^{2}$, and Mandana Ahmadi ${ }^{1}$ \\ 1 Nordita, Blegdamsvej 17, DK-2100 Copenhagen $\varnothing$, \\ hertz@nordita.dk, \\ WWW home page: http://www.nordita.dk/ hertz \\ 2 Ørsted-DTU, Technical University of Denmark, \\ DK-2800 Lyngby
}

\begin{abstract}
We review the use of mean field theory for describing the dynamics of dense, randomly connected cortical circuits. For a simple network of excitatory and inhibitory leaky integrate-and-fire neurons, we can show how the firing irregularity, as measured by the Fano factor, increases with the strength of the synapses in the network and with the value to which the membrane potential is reset after a spike. Generalizing the model to include conductance-based synapses gives insight into the connection between the firing statistics and the high-conductance state observed experimentally in visual cortex. Finally, an extension of the model to describe an orientation hypercolumn provides understanding of how cortical interactions sharpen orientation tuning, in a way that is consistent with observed firing statistics.
\end{abstract}

\section{Introduction}

Neocortical circuits are highly connected: a typical neuron receives synaptic input from of the order of 10000 other neurons. This fact immediately suggests that mean field theory should be useful in describing cortical network dynamics. Furthermore, a good fraction, perhaps half, of the synaptic connections are local, from neurons not more than half a millimeter away, and on this length scale (i.e., within a "cortical column") the connectivity appears to be highly random, with a connection probability of the order of $10 \%$. This requires a level of mean field theory a step beyond the kind used for uniform systems in condensed matter physics like ferromagnets. It has to describe correctly the fluctuations in the inputs to a given network element as well as their mean values, as in spin glasses. The theory we use here is, in fact, adapted directly from that for spin glasses.

A generic feature of mean field theory for spin glasses and other random systems is that the "quenched disorder" in the connections (the connection strengths in the network do not vary in time) leads to an effectively noisy input to a single unit that one studies: Spatial disorder is converted to temporal. The presence of this noise offers a fundamental explanation for the strong irregularity of firing observed experimentally in cortical neurons. For high connectivity, the noise is 
Gaussian, and the correct solution of the problem requires its correlation function to be found self-consistently. In this paper we summarize how to do this for some simple models for cortical networks.

We focus particularly on the neuronal firing statistics. There is a long history of experimental investigations of the apparent noisiness of cortical neurons [719], but very little in the way of theoretical work based on network models. Our work begins to fill that gap in a natural way, since the full mean field theory of a random network is based on self-consistently calculating the correlation function. In particular, we are able to identify the features of the neurons and synapses in the network that control the firing correlations.

The basic ideas developed here were introduced in a short paper [1], and these models are treated in greater detail in several other papers [2-6]. Here we just want to give a quick overview of the mean field approach and what we can learn from it.

\section{Single column model, current-based synapses}

In all the work described here, our neurons are of the leaky integrate-and-fire kind, though it is straightforward to extend the method to other neuronal models, based, for example, on Hodgkin-Huxley equations. In our simplest model, we consider networks of excitatory and inhibitory neurons, each of which receives a synaptic connection from every other neuron with the same probability. Each such connection has a "strength," the amount by which a presynaptic spike changes the postsynaptic potential. In this model, these strengths are independent of the postsynaptic membrane potential ("current-based synapses"). All excitatory-to-excitatory connections that are present are taken to have the same strength, and analogously for the three other classes of connections (excitatoryto-inhibitory, etc.). However, the strengths for the different classes are not the same. In addition, excitatory and inhibitory neurons both receive excitation from an external population, representing "the rest of the brain". (For primary sensory cortices, this excitation includes the sensory input from the thalamus.) This is probably the simplest generic model for a generic "cortical column" of spiking neurons.

The network is taken to have $N_{1}$ excitatory and $N_{2}$ inhibitory neurons. A given neuron (of either kind) receives synaptic input from every excitatory (resp. inhibitory) neuron with probability $K_{1} / N_{1}\left(\right.$ resp. $\left.K_{2} / N_{2}\right)$, with $K_{a} / N_{a}$ independent of $a$. In our calculations we take the connection density $K_{a} / N_{a}$ to be $10 \%$, but the results are not very sensitive to its value as long as it is fairly small. Each nonzero synapse from a neuron in population $b$ to one in population $a$ is taken to have the value $J_{a b} / \sqrt{K_{b}}$. Synapses from the external population are treated in the same way, with strengths $J_{a 0} / \sqrt{K_{0}}$. For simplicity, neurons in the external population are assumed to fire like stationary independent Poisson

processes. We consider the limit $K_{a} \rightarrow \infty, N_{a} \rightarrow \infty$, with $K_{a} / N_{a}$ fixed, where mean field theory is exact. 
The subthreshold dynamics of the membrane potential of neuron $i$ in population $a$ obey

$$
\frac{\mathrm{d} u_{i}^{a}}{\mathrm{~d} t}=-\frac{u_{i}^{a}}{\tau}+\sum_{b=0}^{2} \sum_{j=1}^{N_{b}} J_{i j}^{a b} S_{j}^{b}(t),
$$

where $S_{j}^{b}(t)=\sum_{s} \delta\left(t-t_{j s}^{b}\right)$ is the spike train of neuron $j$ in population $b$. The membrane time constant is taken to have the same value $\tau$ for all neurons. We give the firing thresholds a narrow distribution of values (10\% of the mean value, 1). We take the firing thresholds $\theta_{a}=1$ and the postfiring reset levels to be 0 . We ignore transmission delays.

The essential point of mean field theory is that for such a large, homogeneously random network, as for an infinite-range spin glass [20-22], we can treat the net input to a neuron as a Gaussian random process. This reduces the network problem to a single-neuron one, with the feature that the statistics of the input have to be determined self-consistently from the firing statistics of the single neurons. This reduction was proved formally for a network of spiking neurons by Fulvi Mari [23].

Explicitly, the effective input current to neuron $i$ in population $a$ can be written

$$
I_{i}^{a}(t)=\sum_{b} J_{a b}\left[\sqrt{K_{b}} r_{b}+B_{b} x_{i}^{a b}+\sqrt{1-K_{b} / N_{b}} \xi_{i}^{a b}(t)\right] .
$$

Here $r_{b}=N_{b}^{-1} \sum_{j} r_{j}^{b}$ is the average rate in population $b$,

$$
B_{b}=\sqrt{\left(1-\frac{K_{b}}{N_{b}}\right) \overline{\left(r_{j}^{b}\right)^{2}}},
$$

$x_{i}^{a b}$ is a unit-variance Gaussian random number, and $\xi_{i}^{a b}(t)$ is a (zero-mean) Gaussian noise with correlation function equal to $C_{b}\left(t-t^{\prime}\right)$, the average correlation function in population $b$. For the contribution from a single population, labeled by $b$, the first term in (2), which represents the mean input current, is larger than the other two, which represent fluctuations, by a factor of $\mathcal{O}\left(\sqrt{K_{b}}\right)$ : averaging over many independent input neurons reduces fluctuations relative to those in a single neuron by the square root of the number of terms in the sum. (For our way of scaling the synapse strengths, the factor $\sqrt{K_{b}}$ in the first term arises formally from adding $K_{b}$ terms, each of which is proportional to $1 / \sqrt{K_{b}}$.)

However, while the fluctuation terms are small in comparison to the mean for a given input population $b$, small compared to the population-averaged input (the first term in (2)), we will see that when we sum over all populations the first term will vanish to leading order. What remains of it is only of the same order as the fluctuations. Therefore fluctuations can not be neglected.

The fact that the fluctuation terms are Gaussian variables is just a consequence of the central limit theorem, since we consider the limit $K_{b} \rightarrow \infty$.

Note that one fluctuation term is static and the other dynamic. The origin of the static one is the fact that the network is inhomogeneous, so different neurons 
will have different number of synapses and therefore different strengths of net time-averaged inputs.

It is perhaps not immediately obvious, but the formal derivation ([23], see also [24] for an analogous case) shows that the dynamic noise also originates from the random inhomogeneity in the network. It would be absent if there were no randomness in the connections, as, for example, in a model like ours but with full connectivity. The presence of the factor $\sqrt{1-K_{a} / N_{a}}$ in the third term in (2) makes this point evident; in the general case the noise variance is proportional to the variance of the connection strengths.

\section{The mean field ansatz}

In any mean field theory, whether it is for a ferromagnet, a superconductor, electroweak interactions, or a neural network, one has to make an ansatz describing the state in question. This ansatz contains some parameters (generally called "order parameters"), the values of which are then determined self-consistently. Here, our "order parameters" are the mean rates $r_{b}$, their mean square values (which appear in (3), and the correlation functions $C_{b}\left(t-t^{\prime}\right)$. We make an ansatz for the correlation functions that describes an asynchronous irregular firing state: We take $r_{b}$ to be time-independent and $C_{b}\left(t-t^{\prime}\right)$ to have a delta-function peak (of strength equal to $r_{b}$ ) at $t=t^{\prime}$, plus a continuous part that falls off toward zero as $\left|t-t^{\prime}\right| \rightarrow \infty$. We could also look, for example, for solutions in which $r_{b}$ was time-dependent and/or $C_{b}\left(t-t^{\prime}\right)$ had extra delta-function peaks (these might describe oscillating population activity ), but we have not done so. Thus, we cannot exclude the existence of such exotic states, but we can at least check whether our asynchronous, irregularly-firing states exist and are stable.

We can find the mean rates, at least when they are low, independently of their fluctuations and the correlation functions: In an irregularly-firing state the membrane potential should fluctuate around a stationary value, with the noise occasionally and irregularly driving it up to threshold. In mean field theory, we have

$$
\frac{\mathrm{d} u_{a}}{\mathrm{~d} t}=-\frac{u_{a}}{\tau}+I_{a}(t)
$$

where $I_{a}(t)$ is given by (2). (We have dropped the neuron index $i$, since we are now doing a one-neuron problem.) From (2), we see that the leading terms in $I_{a}(t)$ are large $\left(\propto \sqrt{K_{b}}\right)$, so if the membrane potential is to be stationary they must nearly cancel:

$$
\sum_{b=0}^{2} J_{a b} \sqrt{K_{b}} r_{b}=\mathcal{O}(1)
$$

That is, the mean excitatory $(b=0,1)$ and inhibitory $(b=2)$ currents must nearly balance. Therefore we call (5) the balance condition. Defining $\hat{J}_{a b}=$ $J_{a b} \sqrt{K_{b} / K_{0}}$, we can also write it in the form

$$
\sum_{b=0}^{2} \hat{J}_{a b} r_{b}=0
$$


The external rate $r_{0}$ is assumed known, so these two linear equations can be solved for $r_{b}, b=1,2$. We can write the solution as

$$
r_{a}=-\sum_{b=1}^{2}\left[\hat{\mathrm{J}}^{-1}\right]_{a b} J_{b 0} r_{0},
$$

where by $\hat{\jmath}^{-1}$ we mean the inverse of the $2 \times 2$ matrix with elements $\hat{J}_{a b}, a, b=$ 1,2. This result was obtained some time ago by Amit and Brunel [25-27] and, for a nonspiking neuron model, by van Vreeswijk and Sompolinsky [28, 29].

However, a complete mean field theory involves the rate fluctuations within the populations and the correlation functions, and it is clear that if we want to understand something quantitative about the degree of irregularity of the neuronal firing, it is necessary to do the full theory. This cannot be done analytically, so we resort to numerical calculation.

\section{Numerical procedure}

Our method was inspired by the work of Eisfeller and Opper [30] on spin glasses. They, too, had a mean field problem that could not be solved analytically, so they solved numerically the single-spin problem to which mean field theory reduced their system. In our case, we have to solve numerically, the problem of a single neuron driven by Gaussian random noise, and the crucial part is to make the input noise statistics consistent with the output firing statistics.

This requires an iterative procedure. We have to start with a guess about the mean rates, the rate fluctuations, and the correlation functions for the neurons in the two populations. We then generate noise according to (2) and simulate many trials of neurons driven by realizations of this noise. In these trials, the effective numbers of inputs $K_{b}$ are varied randomly from trial to trial, with a Gaussian distribution of width $\sqrt{K_{b}}$, to capture the effects of the random connectivity in the network. We compute the firing statistics for these trials and use the result to improve our estimate of the input noise statistics. We then repeat the trials and iterate the loop until the input and output statistics agree.

We can get a good initial estimate of the mean rates from the balance condition equation (7), but this is harder to do for the rate fluctuations and correlation function. The method we have used is to do the initial trials with white noise input (of a strength determined by the mean rates). There seems to be no problem converging to a solution with self consistent rates, rate fluctuations and firing correlations from this starting point.

More details of the procedure can be found in [2].

\section{Some results}

As a measure of the firing irregularity, we consider the Fano factor $F$. It is defined as the ratio of the variance of the spike count to its average, where both statistics are computed over a large number of trials. It is easy to relate it to the correlation function, as follows. 


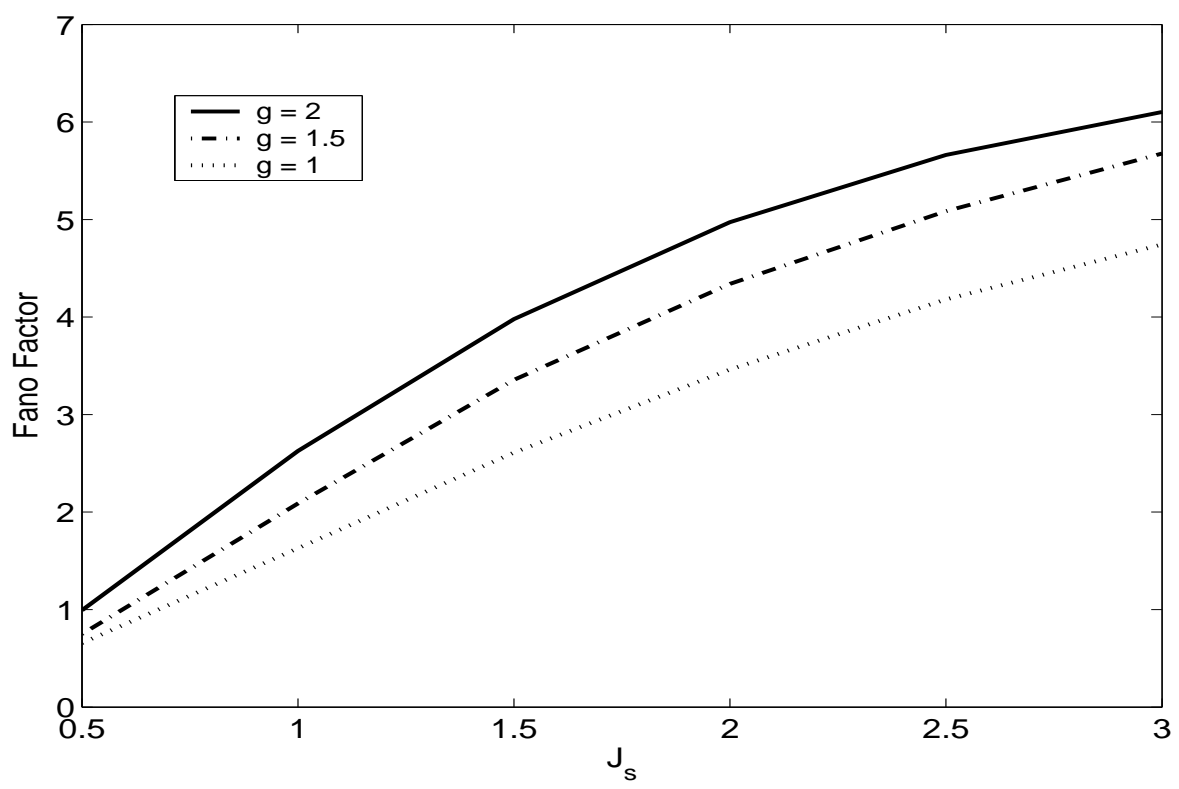

Fig. 1. Fano factor as a function of the overall synaptic scaling parameter $J_{s}$, for 3 values of the relative inhibition parameter $g$.

If $S(t)$ is a spike train as in (1), the spike count in an interval from 0 to $T$ is

$$
n=\int_{0}^{T} \mathrm{~d} t S(t)
$$

Its mean is just $\bar{n}=\int_{0}^{T} r \mathrm{~d} t=r T$, and its variance is

$$
\overline{(n-\bar{n})^{2}}=\int_{0}^{T} \mathrm{~d} t \int_{0}^{T} \mathrm{~d} t^{\prime}\left\langle[S(t)-r)\left(S\left(t^{\prime}\right)-r\right]\right\rangle
$$

The quantity in the averaging brackets in (9) is just the correlation function. Changing integration variables from $\left(t, t^{\prime}\right)$ to $\left(\bar{t}=\frac{1}{2}\left(t+t^{\prime}\right), s=t-t^{\prime}\right)$ and taking $T \rightarrow \infty$ gives

$$
F=1+\frac{1}{r} \int_{-\infty}^{\infty} \mathrm{d} s C(s)
$$

For a Poisson process, $C(s)=r \delta(s)$, leading to $F=1$.

Thus, a Fano factor greater than 1 is not really "more irregular than a Poisson process", since any deviation of $F$ from 1 comes from some kind of firing correlations.

For this model we have studied how the magnitude of the synaptic strengths affects the Fano factor. We have used

$$
\hat{\mathrm{j}}=J_{s}\left(\begin{array}{l}
1-2 g \\
2-2 g
\end{array}\right) .
$$


In Fig. 1 we plot $\mathrm{F}$ as a function of the overall scaling factor $J_{s}$ for three different values of the relative inhibition strength $g$. Evidently, increasing synaptic strength in either way increases $F$.

How can we understand this result? Let us think of the stochastic dynamics of the membrane potential $u$ after a spike and reset, as described, for example, by a Fokker-Planck equation. Right after reset, the distribution of $u$ is a deltafunction at the reset level. Then it spreads out diffusively and its center drifts toward a quasi-equilibrium level. The speed of the spread and the width of the quasi-equilibrium distribution reached after a time $\sim \tau$ are both proportional to the synaptic strength.

This distribution is only "quasi-equilibrium" because on the somewhat longer timescale of the typical interspike interval, significant weight will reach the absorbing boundary at threshold. Nevertheless, we can regard it as nearly stationary if the rate is much less than $\tau^{-1}$.

The center of the quasi-equilibrium distribution has to be at least a standard deviation or so below threshold if the neuron is going to fire at a low-to-moderate rate. Thus, since this width is proportional to the synaptic strengths, if we fix the reset at zero and the threshold at 1 the drift of the distribution after reset will be upward for sufficiently weak strengths and downward for strong enough ones. Hence, in the weak case, there is a reduced probability of spikes (relative to a Poisson process) for times shorter than $\tau$, leading to a refractory dip in the correlation function and a Fano factor bigger than 1. In the strong-synapse case, the rapid initial spread of the membrane potential distribution before it has a chance to drift very far downward leads to excess early spikes, a positive correlation function at short times, and a Fano factor bigger than 1 . The relevant ratio is the width of the quasi-equilibrium membrane potential distribution (for this model, roughly speaking, $J_{s}$ ) divided by the different between reset and threshold.

The above argument applies even for neurons with white noise input. But in the mean field description the firing correlation induced by this effect lead to correlations in the input current, which amplify the effects.

\section{Model with conductance-based synapses}

In a second model, we add a touch of realism, replacing the current-based synapses by conductance-based ones. Then the postsynaptic potential change produced by a presynaptic spike is equal to a strength parameter multiplied by the difference between the postsynaptic membrane potential and the reversal potential for the class of synapse in question. In addition, we include a simple model for synaptic dynamics: we need no longer assume that the postsynaptic potential changes instantaneously in response to the postsynaptic spike.

Now the subthreshold membrane potential dynamics become

$$
\frac{\mathrm{d} u_{i}^{a}(t)}{\mathrm{d} t}=-g_{L} u_{i}^{a}(t)-\sum_{b=0}^{2} \sum_{j=1}^{N_{b}} g_{a b}^{i j}(t)\left(u_{i}^{a}(t)-V_{b}\right)
$$


Here $g_{L}$ is a nonspecific leakage conductance (taken in units of inverse time); it corresponds to $\tau^{-1}$ in (1). The $V_{b}$ are the reversal potentials for the synapses from population $b$; they are above threshold for excitatory synapses and below 0 for inhibitory ones, so the synaptic currents are positive (i.e., inward) and negative (outward), respectively, in these cases. The time-dependent synaptic conductances $g_{a b}^{i j}(t)$ reflect the firing of presynaptic neuron $j$ in population $b$, filtered at its synapse to postsynaptic neuron $i$ in population $a$ :

$$
g_{a b}^{i j}(t)=\frac{g_{a b}^{0}}{\sqrt{K_{b}}} \int_{-\infty}^{t} \mathrm{~d} t^{\prime} K\left(t-t^{\prime}\right) S_{j}\left(t^{\prime}\right)
$$

when a connection between these neurons is present; otherwise it is zero. (We assume the same random connectivity distribution as in the previous model.)

We have taken the synaptic filter kernel $K(t)$ to have the simple form

$$
K(t)=\frac{\mathrm{e}^{-t / \tau_{2}}-\mathrm{e}^{-t / \tau_{1}}}{\tau_{2}-\tau_{1}},
$$

representing an average temporal conductance profile following a presynaptic spike, with characteristic opening and closing times $\tau_{1}$ and $\tau_{2}$. This kernel is normalized so that $\int \mathrm{d} t K(t)=1$; thus, the total time integral of the conductance over the period after an isolated spike is equal to $g_{a b}^{0} / \sqrt{K_{b}}$. Hence, for very short synaptic filtering times, this model looks like (1) with a membrane potential-dependent $J_{i j}^{a b}$ equal to $g_{a b}^{0}\left(u_{a}^{i}(t)-V_{b}\right) / \sqrt{K_{b}}$. We take the (dimensionless) parameters $g_{a b}^{0}$, like the $J_{a b}$ in the previous model, to be of order 1 , so we anticipate a large $\left(\mathcal{O}\left(\sqrt{K_{b}}\right)\right.$ ) mean current input from each population $b$ and, in the asynchronously-firing steady state, a near cancellation of these separately large currents.

In mean field theory, we have the effective single-neuron equation of motion

$$
\frac{\mathrm{d} u_{a}(t)}{\mathrm{d} t}=-g_{L} u_{a}(t)-\sum_{b=0}^{2} g_{a b}(t)\left(u_{a}(t)-V_{b}\right),
$$

in which the total effect of population $b$ on a neuron in population $a$ is a timedependent conductance $g_{a b}(t)$ consisting of a population mean

$$
\overline{\left\langle g_{a b}\right\rangle}=\sqrt{K_{b}} g_{a b}^{0} r_{b},
$$

static noise of variance

$$
\overline{\left(\delta\left\langle g_{a b}\right\rangle\right)^{2}}=\left(1-\frac{K_{b}}{N_{b}}\right)\left(g_{a b}^{0}\right)^{2} \overline{\left(\delta r_{b}\right)^{2}},
$$

and dynamic noise with correlation function

$$
\left\langle\delta g_{a b}(t) \delta g_{a b}\left(t^{\prime}\right)\right\rangle=\left(1-\frac{K_{b}}{N_{b}}\right)\left(g_{a b}^{0}\right)^{2} \tilde{C}_{b}\left(t-t^{\prime}\right),
$$


where

$$
\tilde{C}_{b}\left(t-t^{\prime}\right)=\int_{-\infty}^{t} \mathrm{~d} t_{1} K\left(t-t_{1}\right) \int_{-\infty}^{t^{\prime}} \mathrm{d} t_{2} K\left(t^{\prime}-t_{2}\right) C_{b}\left(t_{1}, t_{2}\right)
$$

is the correlation function of the synaptically filtered spike trains of population $b$.

\section{The balance condition}

As for the model with current-based synapses, we can argue that in an irregularly, asynchronously-firing state the average $\mathrm{d} u_{i}^{a} / \mathrm{d} t$ should vanish. From (15) we obtain

$$
g_{L} \overline{u_{a}}+\sum_{b} g_{a b}^{0} \sqrt{K_{b}} r_{b}\left(\overline{u_{a}}-V_{b}\right)=0 .
$$

Again, for large connectivity the leakage term can be ignored. In contrast to what we found in the current-based case, now the balance condition requires knowing the mean membrane potential $\overline{u_{a}}$. However, we will see that in the mean field limit the membrane potential has a narrow distribution centered just below threshold. Since the fluctuations are very small, the factor $u_{a}-V_{b} \approx \theta_{a}-V_{b}$ in (12) can be regarded as constant, and we are effectively back to the currentbased model. Thus, defining

$$
J_{a b}^{\mathrm{eff}}=g_{a b}^{0}\left(V_{b}-\theta_{a}\right),
$$

we can just apply the analysis from the current-based case.

\section{High-conductance state}

It is useful to measure the membrane potential relative to $\overline{u_{a}}$. So, writing $u_{a}=$ $\overline{u_{a}}+\delta u_{a}(t)$ and using the balance condition (20), we find

$$
\frac{\mathrm{d} \delta u_{a}}{\mathrm{~d} t}=-g_{\mathrm{tot}}(t) \delta u_{a}+\sum_{b} \delta g_{a b}(t)\left(V_{b}-\overline{u_{a}}\right),
$$

where

$$
\begin{aligned}
g_{\mathrm{tot}}(t) & =g_{L}+\sum_{b} g_{a b}(t) \\
& =g_{L}+\sum_{b}\left[\sqrt{K_{b}} g_{a b}^{0} r_{b}+\delta g_{a b}(t)\right],
\end{aligned}
$$

with $\delta g_{a b}(t)$ the fluctuating parts of $g_{a b}(t)$, the statistics of which are given by (17) and (18). This looks like a simple leaky integrator with current input $\sum_{b} \delta g_{a b}(t)\left(V_{b}-\overline{u_{a}}\right)$ and a time-dependent effective membrane time constant equal to $g_{\text {tot }}(t)^{-1}$. Following Shelley et al. [31], (22) can be further rearranged into the form

$$
\frac{\mathrm{d} \delta u_{a}}{\mathrm{~d} t}=-g_{\mathrm{tot}}(t)\left[\delta u_{a}-\delta V_{a}^{s}(t)\right],
$$




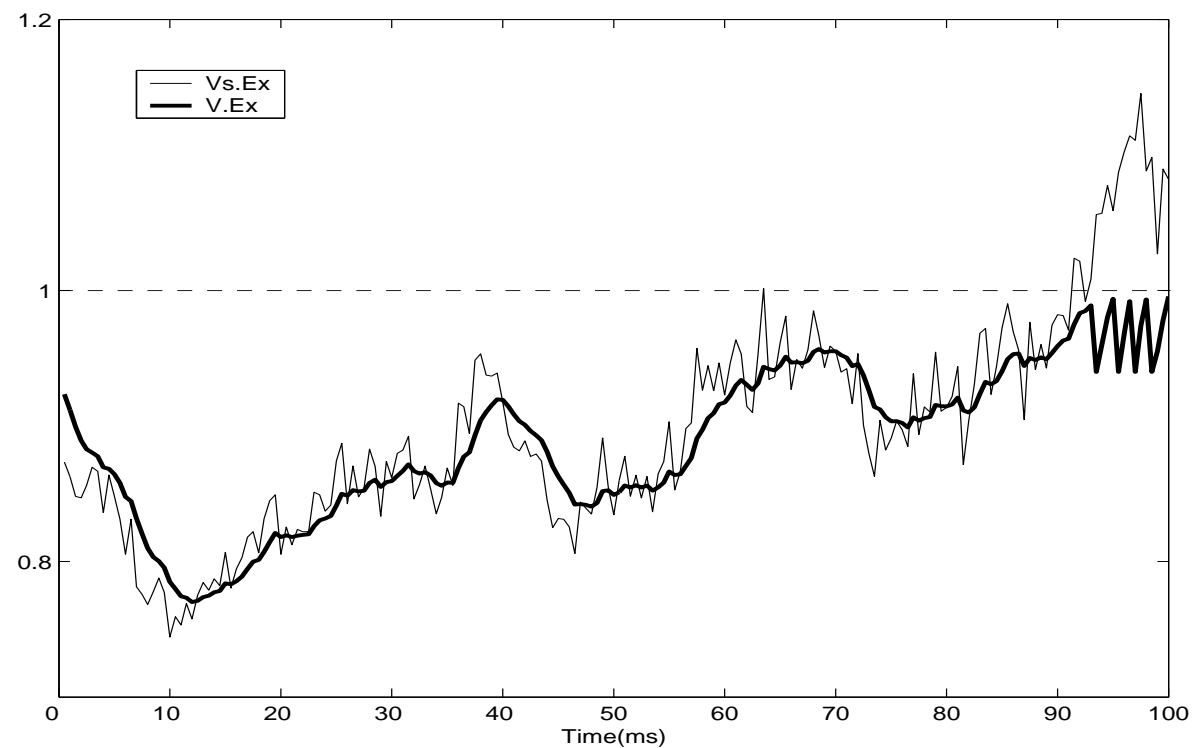

Fig. 2. The membrane potential $u(t)$ follows the effective reversal potential $V_{S}(t)$ closely, except when $V_{s}$ is above threshold. Here, the threshold is 1 and the reset 0.94 .

with the "instantaneous reversal potential" (here measured relative to $\overline{u_{a}}$ ) given by

$$
\delta V_{a}^{s}(t)=\frac{\sum_{b} \delta g_{a b}(t)\left(V_{b}-\overline{u_{a}}\right)}{g_{\mathrm{tot}}(t)} .
$$

Eq. (25) says that at any instant of time, $\delta u_{a}$ is approaching $\delta V_{a}^{s}(t)$ at a rate $g_{\text {tot }}(t)$.

For large $K_{b}, g_{\text {tot }}$ is large $\left(\mathcal{O}\left(\sqrt{K_{b}}\right)\right)$, so the effective membrane time constant is very small and the membrane potential follows the fluctuating $\delta V_{a}^{s}(t)$ very closely. Fig. 2 shows an example from one of our simulations. This is the main qualitative difference between mean field theory for the model with currentbased synapses and the one with conductance-based ones. It is also the reason we introduced synaptic filtering into the present model. In the current-based one, the membrane potential filtered the input current with a time constant $\tau$ which we could assume to be long compared with synaptic time constants, so we could safely ignore the latter. But here the effective membrane time constant becomes shorter than the synaptic filtering times, so we have to retain the kernel $K(t) 14)$. Here we have argued this solely from the fact that we are dealing with the mean field limit, but Shelley et al. argue that it actually applies to primary visual cortex (see also [32]).

We also observe that in the mean field limit, both the leakage conductance and the fluctuation term $\delta g(a b(t)$ are small in comparision with the mean, so we 
can approximate $g_{t o t}(t)$ by a constant:

$$
g_{\mathrm{tot}}=\sum_{b} \sqrt{K_{b}} g_{a b}^{0} r_{b}
$$

Furthermore, the fluctuations $\delta V_{a}^{s}(t)$ in the instantaneous reversal potential (26) are then of order $1 / \sqrt{K_{b}}$ : membrane potential fluctuations can not go far from $\overline{u_{a}}$. But $V_{a}^{s}(t)$ must go above threshold frequently enough to produce firing at the self-consistent rates. Thus, $\overline{u_{a}}$ must lie just a little below threshold, as promised above. Hence, at fixed firing rates, the conductance-based problem effectively reduces to a current-based one with a very small effective membrane time constant $\tau_{\text {eff }}=g_{\text {tot }}^{-1}$ and synaptic coupling parameters $J_{a b}^{\text {eff }}$ given by $(21)$. Of course, as we increase the firing rates of the external population and thereby increase the rates in the network, we will change $g_{\text {tot }}$, making both $\tau_{\text {eff }}$ and the fluctuations $\delta V_{a}^{s}(t)$ correspondingly smaller.

If we neglected synaptic filtering, the resulting dynamics would be rather trivial. It would be self-consistent to take the input current as essentially white noise, for then excursions of $\delta V_{a}^{s}(t)$ above threshold would be be uncorrelated, and, since the membrane potential could react instantaneously to follow it up to threshold, so would the firing be. (Simulations confirm this argument.)

Therefore, the synaptic filtering is essential. It imparts a positive correlation time to the fluctuations $\delta V_{a}^{s}(t)$, so if it rises above threshold it can stay there for a while. During this time, the neuron will fire repeatedly, leading to a positive tail in the correlation function for times of the order of the synaptic time constants. This broader the kernel $K\left(t-t^{\prime}\right)$, the stronger this effect. In the self-consistent description, this effect feeds back on itself: $\delta V_{a}^{s}(t)$ acquires even longer correlations, and these lead to even stronger bursty firing correlations.

Thus, the mean field limit $K_{b} \rightarrow \infty$ can be pathological in the conductancebased model with synaptic filtering. However, here we take the view that mean field theoretical calculations may still give a useful description real cortical dynamics, despite that fact that real cortex is not described by the $K_{b} \rightarrow \infty$ limit. For example, the true effective membrane time constant is not zero, but, according to experiment [32], it is significantly reduced from its in vitro value by synaptic input, probably [31] to a value less than characteristic synaptic filtering times. Doing mean field theory with moderately, but not extremely large connectivities can describe such a state in a natural and transparent way.

As in the current-based model, the Fano factor grows with the ratio of the membrane potential distribution to the threshold-reset difference. It also grows with increasing synaptic filtering time, as argued above. Fig. 3 shows $F$ plotted as a function of $\tau_{2}$, with $\tau_{1}$ fixed at $1 \mathrm{~ms}$, for a set of reset values.

\section{Orientation hypercolumn model}

Finally, we try to take a step beyond homogeneously random models to describe networks with systematic structure in their connections. We consider the example of a hypercolumn in primary visual cortex: a collection of $n$ orientation 


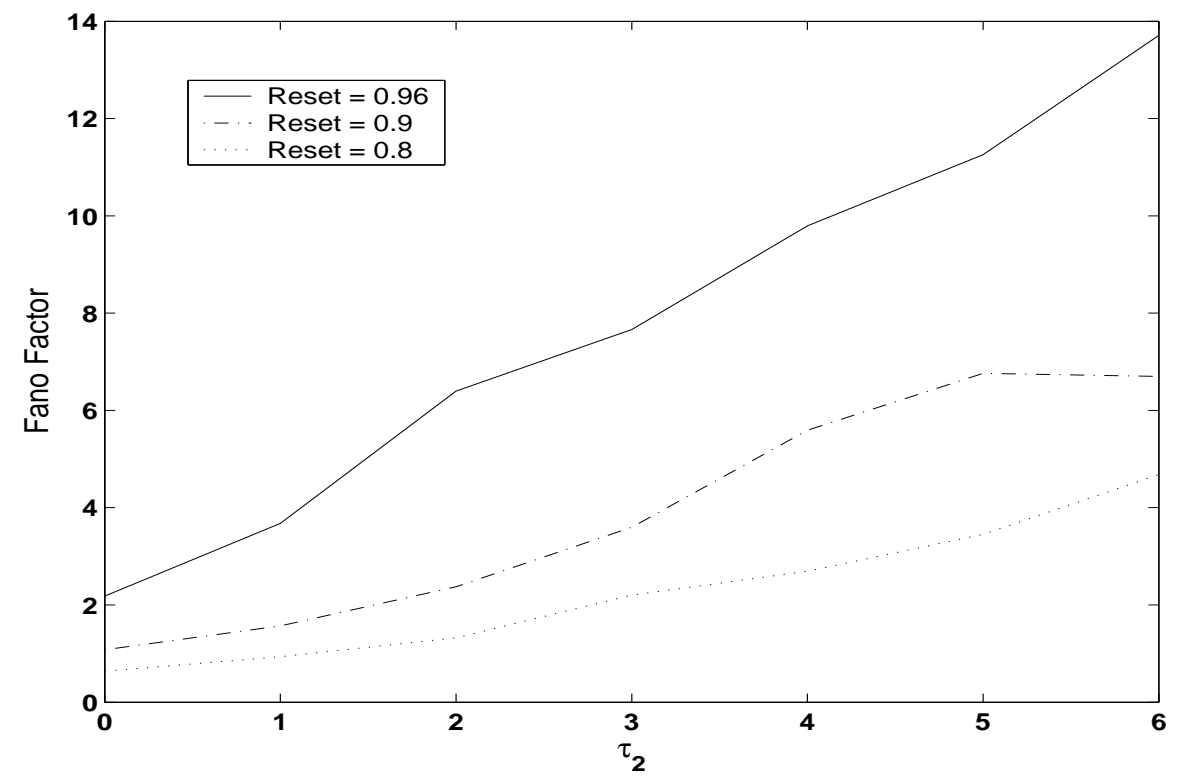

Fig. 3. Fano factor as a function of $\tau_{2}$ for 3 reset values.

columns, within which each neuron responds most strongly to a stimulus of a particular orientation. The hypercolumn contains columns that cover the full range of possible stimulus orientations from 0 to $\pi$. It is know that columns with similar orientation selectivities interact more strongly than those with dissimilar ones (because they tend to lie closer to each other on the cortical surface). We build this structure into an extended version of our model, which can be treated with essentially the same mean field methods as the simpler, homogeneously random one. In the version we present here, we revert to current-based synapses, but it is straightforward to construct a corresponding model with conductance-based ones.

A study of similar model, for non-spiking neurons, was reported by Wolf et al. [33]. Those authors have also simulated a network of spiking neurons like the one described here [34], complementing the mean-field analysis we give here.

Each neuron now acquires an extra index $\theta$ labeling the stimulus orientation to which it responds most strongly, so the equations of motion for the membrane potentials become

$$
\frac{\mathrm{d} u_{i}^{a \theta}}{\mathrm{d} t}=-\frac{u_{i}^{a \theta}}{\tau}+I_{i}^{a}\left(\theta, \theta_{0}, t\right)+\sum_{b=1}^{2} \sum_{\theta^{\prime}=1}^{n} \sum_{j=1}^{N_{b} / n} J_{i j}^{a \theta, b \theta^{\prime}} S_{j}^{b \theta}(t) .
$$

The term $I_{i}^{a}\left(\theta, \theta_{0}, t\right)$ represents the external input current for a stimulus with orientation $\theta_{0}$. (In this section we set all thresholds equal to 1 , and $\theta$ refers only to orientation.) We assume it comes through diluted connections from a 
population of $N_{0} \gg 1$ Poisson neurons which fire at a constant rate $r_{0}$ :

$$
I_{i}^{a}\left(\theta, \theta_{0}, t\right)=\sum_{j} J_{i j}^{a 0}\left(\theta, \theta_{0}\right) S_{j}^{0}(t)
$$

As in the single-column model, we take the nonzero connections to have the value

$$
J_{i j}^{a 0}\left(\theta, \theta_{0}\right)=\frac{J_{a 0}}{\sqrt{K_{0}}}
$$

but now we take the connection probability to depend on the difference between $\theta$ and $\theta_{0}$, according to

$$
P_{0}\left(\theta, \theta_{0}\right)=\frac{K_{0}}{N_{0}}\left[1+\epsilon \cos 2\left(\theta-\theta_{0}\right)\right] .
$$

This tuning is assumed to come from a Hubel-Wiesel feed-forward connectivity mechanism. The general form has to be periodic with period $\pi$ and so would have terms proportional to $\cos 2 m\left(\theta-\theta_{0}\right)$ for all $m$, but here, following Ben-Yishai et $a l$. [35], we use the simplest form possible. We have also assumed that the degree of tuning, measured by the anisotropy parameter $\epsilon$, is the same for inhibitory and excitatory postsynaptic neurons.

Assuming isotropy, we are free to measure all orientations relative to $\theta_{0}$, so we set $\theta_{0}=0$ from now on.

Similarly, we take the nonzero intracortical interactions $J_{i j}^{a \theta, b \theta^{\prime}}$ to be

$$
J_{i j}^{a \theta, b \theta^{\prime}}=\frac{J_{a b}}{\sqrt{K_{b}}}
$$

and take the probability of connection to be

$$
P_{a b}\left(\theta-\theta^{\prime}\right)=\frac{K_{b}}{N_{b}}\left[1+\gamma \cos 2\left(\theta-\theta^{\prime}\right)\right] .
$$

Analogously to (31), $P_{a b}$ is independent of both the population indices $a$ and $b$, since we always take $K_{b} / N_{b}$ independent of $b$.

In real cortex, cells are arranged so they generally lie closer to ones of similar than to ones of dissimilar orientation preference, and they are more likely to have connections with nearby cells than with distant ones. This is the anatomy that we model with (33). In [4] and [5] a slightly different model was used, in which the connection probability was taken constant but the strength of the connections was varied like (33). The equations below for the balance condition and the column population rates are the same for both models, but the fluctuations are a little different.

As for the input tuning, the form (33) is just the first two terms in a Fourier series, but again we use the simplest possible form for simplicity. 


\section{Balance condition and solving for population rates}

The balance condition for the hypercolumn model is simply that the net synaptic current should vanish for each column $\theta$. Going over to a continuum notation by writing the sum on columns as an integral, we get

$$
\sqrt{K_{0}} J_{a 0}(1+\epsilon \cos 2 \theta) r_{0}+\sum_{b} \int_{-\pi / 2}^{\pi / 2} \frac{\mathrm{d} \theta^{\prime}}{\pi} J_{a b}\left[1+\gamma \cos 2\left(\theta-\theta^{\prime}\right)\right] \sqrt{K_{b}} r_{b}\left(\theta^{\prime}\right)=0
$$

We have to distinguish two cases: broad and narrow tuning. In the broad case, the rates $r_{b}(\theta)$ are positive for all $\theta$. In the narrowly-tuned case (the physiologically realistic one), $r_{b}(\theta)$ is zero for $|\theta|$ greater than some $\theta_{c}$, which we call the tuning width. (In general $\theta_{c}$ could be different for excitatory and inhibitory neurons, but with our $a$-independent $\epsilon$ in (31) and $a$ - and $b$-independent $\gamma$ in (33), it turns out not to.)

In the broad case the integral over $\theta^{\prime}$ can be done trivially with the help of the trigonometric identity $\cos (A-B)=\cos A \cos B+\sin A \sin B$ and expanding $r_{b}\left(\theta^{\prime}\right)=r_{b, 0}+r_{b, 2} \cos 2 \theta^{\prime}+\cdots$. We find that the higher Fourier components $r_{b, 2 m}, m>1$, do not enter the result:

$$
\sqrt{K_{0}} J_{a 0}(1+\epsilon \cos 2 \theta) r_{0}+\sum_{b} \sqrt{K_{b}} J_{a b}\left(r_{b, 0}+\frac{1}{2} \gamma r_{b, 2} \cos 2 \theta\right)=0 .
$$

If (35) is to hold for every $\theta$, the constant piece and the part proportional to $\cos 2 \theta$ both have to vanish: for each Fourier component we have an equation like (5). Thus we get a pair of equations like (7):

$$
r_{a, 0}=-\sum_{b=1}^{2}\left[\hat{\mathrm{J}}^{-1}\right]_{a b} J_{b 0} r_{0} \quad r_{a, 2}=-\frac{2 \epsilon}{\gamma} \sum_{b=1}^{2}\left[\hat{\mathrm{J}}^{-1}\right]_{a b} J_{b 0} r_{0}\left(=\frac{2 \epsilon}{\gamma} r_{a, 0}\right),
$$

where $\hat{J}_{a b}=J_{a b} \sqrt{K_{b} / K_{0}}$, as in the simple model.

This solution is acceptable only if $\epsilon \leq \gamma / 2$, since otherwise $r_{a}(\theta)$ will be negative for $\theta>\frac{1}{2} \cos ^{-1}\left(-r_{a, 0} / r_{a, 2}\right)$.

Therefore, for $\epsilon>\gamma / 2$, we make the ansatz

$$
r_{a}(\theta)=r_{a, 2}\left(\cos 2 \theta-\cos 2 \theta_{c}\right)
$$

(i.e., we write $r_{a, 0}$ as $-r_{a, 2} \cos 2 \theta_{c}$ ) for $|\theta|<\theta_{c}$ and $r_{a}(\theta)=0$ for $|\theta| \geq \theta_{c}$. We put this into the balance condition (34). Now the integrals run from $-\theta_{c}$ to $\theta_{c}$, so they are as trivial as in the broadly-tuned case, but the ansatz works and we find

$$
J_{a 0} r_{0}+\sum_{b} \hat{J}_{a b} r_{b, 2} f_{0}\left(\theta_{c}\right)=0, \quad \epsilon J_{a 0} r_{0}+\gamma \sum_{b} \hat{J}_{a b} r_{b, 2} f_{2}\left(\theta_{c}\right)=0
$$

where

$$
f_{0}\left(\theta_{c}\right)=\frac{1}{\pi}\left(\sin 2 \theta_{c}-2 \theta_{c} \cos 2 \theta_{c}\right), \quad f_{2}\left(\theta_{c}\right)=\frac{1}{\pi}\left(\theta_{c}-\frac{1}{4} \sin 4 \theta_{c}\right),
$$


(The algebra here is essentially the same as that in a different kind of model studied by Ben-Yishai et al. [35]; see also [36].)

Eqns. (38) can be solved for $\theta_{c}$ and $r_{a, 2}, a=1,2$. Dividing one equation by the other leads to the following equation for $\theta_{c}$ :

$$
\frac{f_{2}\left(\theta_{c}\right)}{f_{0}\left(\theta_{c}\right)}=\frac{\epsilon}{\gamma}
$$

Then one can use either of the pairs of equations (38) to find the remaining unknowns $r_{a, 2}$ :

$$
r_{a, 2}=-\frac{1}{f_{0}\left(\theta_{c}\right)} \sum_{b}\left[\hat{\jmath}^{-1}\right]_{a b} J_{b 0} r_{0} .
$$

The function $f_{2}\left(\theta_{c}\right) / f_{0}\left(\theta_{c}\right)$ takes the value 1 at $\theta_{c}=0$ and falls monotonically to $\frac{1}{2}$ at $\theta_{c}=\pi / 2$. Thus, a solution can be found for $\frac{1}{2} \leq \epsilon / \gamma \leq 1$. For $\epsilon / \gamma \rightarrow \frac{1}{2}$, $\theta_{c} \rightarrow \pi / 2$ and we go back to the broad solution. For $\epsilon / \gamma \rightarrow 1, \theta_{c} \rightarrow 0$ : the tuning of the rates becomes infinitely narrow. Note that stronger tuning of the cortical interactions (bigger $\gamma$ ) leads to broader orientation tuning of the cortical rates. This possibly surprising result can be understood if one remembers that the cortical interactions (which are essentially inhibitory) act divisively (see, e.g., (36) and (41)).

Another feature of the solution is that, from (40), the tuning width does not depend on the input rate $r_{0}$, which we identify with the contrast of the stimulus. Thus, in the narrowly-tuned case, the population rates in this model automatically exhibit contrast-invariant tuning, in agreement with experimental findings [37]. We can see that this result is a direct consequence of the balance condition.

However, we should note that individual neurons in a column will exhibit fluctuations around the mean tuning curves which are not negligible, even in the mean-field limit. These come from the static part of the fluctuations in the input current (like the second term in (2) for the single-column model), which originate from the random inhomogeneity of the connectivity in the network.

As for the single-column model, the full solution, including the determination of the rate fluctuations and correlation functions, has to be done numerically. This only needs a straightforward extension of the iterative procedure described above for the simple model.

\section{Tuning of input noise}

We now consider the tuning of the dynamic input noise. Using the continuum notation, we get input and recurrent contributions adding up to

$$
\begin{aligned}
\left\langle\delta I_{a}(\theta, t) \delta I_{a}\left(\theta, t^{\prime}\right)\right\rangle & =J_{a 0}^{2}(1+\epsilon \cos 2 \theta) r_{0} \delta\left(t-t^{\prime}\right) \\
& +\sum_{b} \int_{-\pi}^{\pi} \frac{\mathrm{d} \theta^{\prime}}{\pi} J_{a b}^{2}\left[1+\gamma \cos 2\left(\theta-\theta^{\prime}\right)\right] C_{b}\left(\theta^{\prime}, t-t^{\prime}\right),
\end{aligned}
$$


where $C_{b}\left(\theta^{\prime}, t-t^{\prime}\right)$ is the correlation function for population $b$ in column $\theta^{\prime}$. We can not proceed further analytically for $t \neq t^{\prime}$, since this correlation function has to be determined numerically. But we know that for an irregularly firing state $C_{b}\left(\theta, t-t^{\prime}\right)$ always has a piece proportional to $r_{b}(\theta) \delta\left(t-t^{\prime}\right)$. This, together with the external input noise, gives a flat contribution to the noise spectrum of

$$
\lim _{\omega \rightarrow \infty}\left\langle\left|\delta I_{a}(\theta, \omega)\right|^{2}\right\rangle=J_{a 0}^{2}(1+\epsilon \cos 2 \theta) r_{0}+\sum_{b} \int_{-\pi}^{\pi} \frac{\mathrm{d} \theta^{\prime}}{\pi} J_{a b}^{2}\left[1+\gamma \cos 2\left(\theta-\theta^{\prime}\right)\right] r_{b}\left(\theta^{\prime}\right) .
$$

The integrals on $\theta^{\prime}$ are of the same kind we did in the calculation of the rates above, so we get

$$
\begin{aligned}
\lim _{\omega \rightarrow \infty}\left\langle\left|\delta I_{a}(\theta, \omega)\right|^{2}\right\rangle & =J_{a 0}^{2}(1+\epsilon \cos 2 \theta) r_{0}+\sum_{b} J_{a b}^{2} r_{b, 2}\left[f_{0}\left(\theta_{c}\right)+\gamma f_{2}\left(\theta_{c}\right) \cos 2 \theta\right] \\
& =r_{0}(1+\epsilon \cos 2 \theta)\left(J_{a 0}^{2}-\sum_{b c} J_{a b}^{2}\left[\hat{\jmath}^{-1}\right]_{b c} J_{c 0}\right)
\end{aligned}
$$

where we have used (40) and (41) to obtain the last line. Thus, the recurrent synaptic noise has the same orientation tuning as that from the external input, unlike the population firing rates, which are narrowed by the cortical interactions.

\section{Output noise: tuning of the Fano factor}

To study the tuning of the noise in the neuronal firing, we have to carry out the full numerical mean field computation. Fig. 4 shows results for the tuning of the Fano factor with $\theta$, for three values of the overall synaptic strength factor $J_{s}$. For small $J_{s}$ there is a minimum in $F$ at the optimal orientation (0), while for large $J_{s}$ there is a maximum. It seems that for any $J_{s}, F$ is either less than 1 at all angles or greater than 1 at all angles; we have not found any cases where the firing changes from subpoissonian to superpoissonian as the orientation is varied.

\section{Discussion}

These examples show the power of mean field theory in studying the dynamics of dense, randomly-connected cortical circuits, in particular, their firing statistics, described by the autocorrelation function and quantities derived from it, such as the Fano factor. One should be careful to distinguish this kind of mean field theory from ones based on "rate models", where a function giving the firing rate as a function of the input current is given by hand as part of the model. By construction, those models can not say anything about firing statistics. Here we are working at a more microscopic level, and both the equations for the firing rates and the firing statistics emerge from a self-consistent calculation. We think it is important to do a calculation that can tell us something about firing 


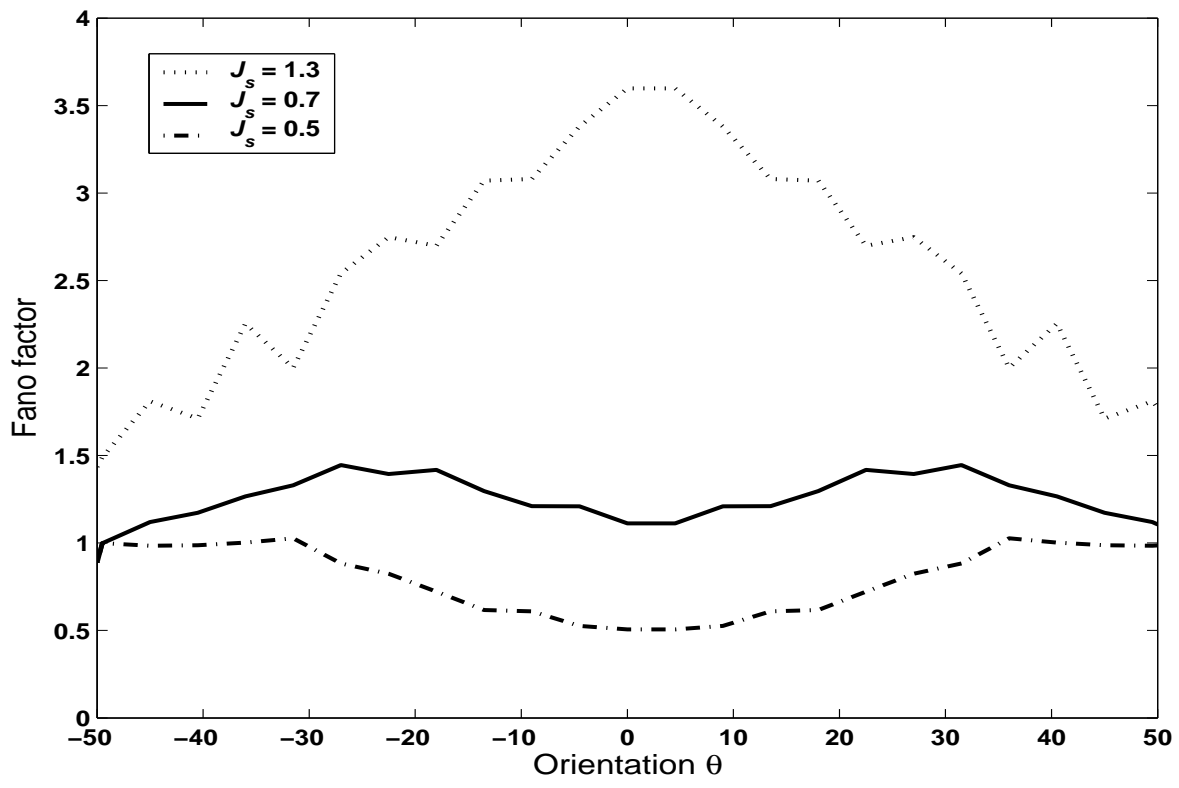

Fig. 4. Fano factor as a function of orientation $\theta$ for 3 values of $J_{s}$.

statistics and correlations, since the irregular firing of cortical neurons is a basic experimental fact that should be explained, preferably quantitatively, not just assumed.

We were able to see in the simplest model described here how this irregularity emerges in mean field theory, provided cortical inhibition is strong enough. This confirms results of [25-27], but extends the description to a fully self-consistent one, including correlations.

It became apparent how the strength of synapses and the post-spike reset level controlled the gross characteristics of the firing statistics, as measured by the Fano factor. A high reset level and/or strong synapses result in an enhanced probability of a spike immediately after reset, leading to a tendency toward bursting. Low reset and/or weak synapses have the opposite effect.

Visual cortical neurons seem to show typical Fano factors generally somewhat above the Poisson value of 1 . They have also been shown to have very high synaptic conductances under visual stimulation. Our mean-field analysis of the model with conductance-based synapses shows how these two observed properties may be connected.

In view of the large variation of Fano factors that there could be, it is perhaps remarkable that observed values do not vary more than they do. We like to speculate about this as a coding issue: Any constrained firing correlations imply reduced freedom to encode input variations, so information transmission capacity is maximized when correlations are minimized. Thus, plausibly, evolutionary pressure can be expected to keep synaptic strengths in the right range. 
Finally, extending the description from a single "cortical column" to an array or orientation columns forming a hypercolumn provided a way of understanding the intracortical contribution to orientation tuning, consistent with the basic constraints of dominant inhibition, irregular firing and high synaptic conductance.

These issues can also be addressed directly with large-scale simulations, as in [31]. However mean field theory can give some clearer insight (into the results of such simulations, as well as of experiments), since it reduces the network problem to a single-neuron one, which we have a better chance of understanding. So we think it is worth doing mean field theory even when it becomes as computationally demanding as direct network simulation (as it does in the case of the orientation hypercolumn [34]). At the least, comparison between the two approaches can allow one to identify clearly any true correlation effects, which are, by definition, not present in mean field theory.

Much more can be done with mean field theory than we have described here. First, as mentioned above, it can be done for any kind of neuron, even a multicompartment one, not just a point integrate-and-fire one. At the level of the connectivity model, the simple model described by (33) can also be extended to include more details of known functional architecture (orientation pinwheels, layered structure, etc.). It is also fairly straightforward to add synaptic dynamics (not just the average description of opening and closing of the channels on the postsynaptic side described by the kernel $K\left(t-t^{\prime}\right)$ in (13)). One just has to add a synaptic model which takes the spikes produced by our single effective neuron as input to a synaptic model. Thus, the path toward including more potentially relevant biological detail is open, at non-prohibitive computational cost.

\section{References}

1. J A Hertz, B J Richmond and K Nilsen: Anomalous response variability in a balanced cortical network. Neurocomputing 52-54 (2003) 787-792

2. A Lerchner, C Ursta, J A Hertz, M Ahmadi and P Ruffiot: Response variability in balanced cortical networks (submitted)

3. A Lerchner, M Ahmadi, and J A Hertz: High conductance states in a mean field cortical model. Neurocomputing (to be published, 2004)

4. G Sterner: Modeling orientation selectivity in primary visual cortex. MS thesis, Royal Institute of Technology, Stockholm (2003)

5. J Hertz and G Sterner: Mean field model of an orientation hypercolumn. Soc Neurosci Abstr 911.19 (2003)

6. A Lerchner, G Sterner, J A Hertz and M Ahmadi: Mean field theory for a balanced hypercolumn model of orientation selectivity in primary visual cortex (submitted)

7. $\mathrm{P}$ Heggelund and $\mathrm{K}$ Albus, Response variability and orientation discrimination of single cells in in striate cortex of cat. Exp Brain Res 32 197-211 (1978)

8. A F Dean, The variability of discharge of simple cells in the cat striate cortex. Exp Brain Res 44 437-440 (1981)

9. D J Tolhurst, J A Movshon and I D Thompson, The dependence of response amplitude and variance of cat visual cortical neurones on stimulus contrast. Exp Brain Res 41 414-419 (1981) 
10. D J Tolhurst, J A Movshon and A F Dean, The statistical reliability of signals in single neurons in cat and monkey visual cortex. Vision Res 23 775-785 (1983)

11. R Vogels, W Spileers and G A Orban, The response variability of striate cortical neurons in the behaving monkey. Exp Brain Res 77 432-436 (1989)

12. R J Snowden, S Treue and R A Andersen, The response of neurons in areas V1 and MT of the alert rhesus monkey to moving random dot patterns. Exp Brain Res 88 389-400 (1992)

13. M Gur, A Beylin and D M Snodderly, Response variability of neurons in primary visual cortex (V1) of alert monkeys. J Neurosci 17 2914-2920 (1997)

14. M N Shadlen and $\mathrm{W} T$ Newsome, The variable discharge of cortical neurons: implications for connectivity, computation, and information coding. J Neurosci 18 3870-3896 (1998)

15. E Gershon, M C Wiener, P E Latham and B J Richmond, Coding strategies in monkey V1 and inferior temporal cortex. J Neurophysiol 79 1135-1144 (1998)

16. P Kara, $\mathrm{P}$ Reinagel and $\mathrm{R} \mathrm{C}$ Reid, Low response variability in simultaneously recorded retinal, thalamic, and cortical neurons. Neuron 27 635-646 (2000)

17. G T Buracas, A M Zador, M R DeWeese and T D Albright, Efficient discrimination of temporal patterns by motion-sensitive neurons in primate visual cortex. Neuron 20 959-969 (1998)

18. D Lee, N L Port, W Kruse and A P Georgopoulos, Variability and correlated noise in the discharge of neurons in motor and parietal areas of primate cortex. J Neurosci 18 1161-1170 (1998)

19. M R DeWeese, M Wehr and A M Zador, Binary spiking in auditory cortex. $J$ Neurosci 23 7940-7949 (2003)

20. H Sompolinsky and A Zippelius: Rlexational dynamics of the Edwards-Anderson model and the mean-field theory of spin glasses. Phys Rev B 25 6860-6875 (1982)

21. K H Fischer and J A Hertz: Spin Glasses (Cambridge Univ Press, 1991)

22. M Mézard, G Parisi and M A Virasoro: Spin Glass Theory and Beyond (World Scientific, 1987)

23. C Fulvi Mari, Random networks of spiking neurons: instability in the xenopus tadpole moto-neuron pattern. Phys Rev Lett 85 210-213 (2000)

24. R Kree and A Zippelius, Continuous-time dynamics of asymmetrically diluted neural networks. Phys Rev A 36 4421-4427 (1987)

25. D Amit and N Brunel, Model of spontaneous activity and local structured activity during delay periods in the cerebral cortex. Cereb Cortex 7 237-252 (1997)

26. D Amit and N Brunel, Dynamics of a recurrent network of spiking neurons before and following learning. Network 8 373-404 (1997)

27. N Brunel, Dynamics of sparsely connected networks of excitatory and inhibitory spiking neurons. J Comput Neurosci 8 183-208 (2000)

28. C van Vreeswijk and H Sompolinsky, Chaos in neuronal networks with balanced excitatory and inhibitory activity. Science 274 1724-1726 (1996)

29. C van Vreeswijk and H Sompolinsky, Chaotic balanced state in a model of cortical circuits. Neural Comp 10 1321-1371 (1998)

30. H Eisfeller and M Opper, New method for studying the dynamics of disordered spin systems without finite-size effects. Phys Rev Lett 68 2094-2097 (1992)

31. M Shelley, D McLaughlin, R Shapley, and J Wielaard, States of high conductance in a large-scale model of the visual cortex. J Comput Neurosci 13 93-109 (2002)

32. A Destexhe and D Paré, Impact of network activity on the integrative properties of neocortical pyramidal neurons in vivo. J Neurophysiol 81 1531-1547 (1999)

33. F Wolf, C van Vreeswijk and H Sompolinsky: Chaotic balanced activity induces contrast invariant orientation tuning. Soc Neurosci Abstr 12.7 (2001) 
34. H Sompolinsky (private communication, 2003)

35. R Ben-Yishai R Lev Bar-Or and H Sompolinsky: Theory of orientation tuning in visual cortex. Proc Nat Acad Sci USA 92 3844-3848 (1995)

36. D Hansel and H Sompolinsky: Modeling feature selectivity in local cortical circuits. in Methods in Neuronal Modeling: from Synapse to Networks, C Koch and I Segev, eds (MIT Press, 1998)

37. G Sclar and R Freeman: Orientation selectivity in cat's striate cortex is invariant with stimulus contrast. Exp Brain Res 46 457-461 (1982) 